\title{
RECENT PROGRESS IN A BEAM-BEAM SIMULATION CODE FOR CIRCULAR HADRON MACHINES
}

\author{
Andreas Kabel, SLAC*; Wolfram Fischer, BNL; Tanaji Sen, FNAL
}

\section{Abstract}

While conventional tracking codes can readily provide higher-order optical quantities and give an estimate of dynamic apertures, they are unable to provide directly measurable quantites such as lifetimes and loss rates. The particle tracking framework $\mathrm{Pl} i \mathrm{bb}$ aims at modelling a storage ring with sufficient accuracy and a sufficiently high number of turns and in the presence of beam-beam interactions to allow for an estimate of these quantities. We provide a description of new features of the codes; we also describe a novel method of treating chromaticity in ring sections in a symplectic fashion.

\section{FRAMEWORK}

The most significant changes in the Plibb framework are (1) the separation into a library and application component. The former contains lightweight classes and functions for matrices, phasespace vectors, differential algebra, particles, particle bunches, statistics, random number generation, parameter file parsing and differential algebra and can be readily utilized by other codes. The latter contains executable programs, the most important being the Lifetimeapplication. It contains a MAD-X compatible parser, algorithms to set up a beamline representations and running a set of particle bunches through it, instrumenting it with loss counters and other statistics. (2) The integration of the NIMZOVICH strong-strong code is described below.

Two minor new components have proven to be especially valuable: (1) a MAD-X compatible parser as well as a compatible set of beamline elements allows for relatively painless interaction with standardized lattice files; all modifications-such as lumping, splitting lattices, and inserting elements-can now done within the code without the need to alter lattice files or to extract information from other tracking codes.

(2) Compile-time polymorphism and class injection is now used throughout the code. This technique allows one to write add a new element type by simply writing a single transformation function. The compiled code then contains separately optimized function objects for transformations of phasespace vectors, particle bunches for tracking, differential algebraic objects etc. A lattice is then represented by a sequence of these objects, it can e.g. track a first-order differential-algebraic object to extract a transfer matrix or a particle bunch to estimate a loss rate without any speed

\footnotetext{
*akabel@slac.stanford.edu. Work supported by Department of Energy contract DE-AC02-76SF00515.
}

impact due to polymorphism.

\section{LOSS RATES AND STATISTICS}

One of the aims of the Plibb framework is the immediate computation of observable quantities such as loss rates and beam lifetimes. These quantities are dominated by the behavior of particles of large amplitude. In previous versions of the code, gaussian distributions of particles of equal weights were used; these were 'hollowed out' in six-dimensional phase space to avoid computation of the uninteresting particle dynamics near the core of the beam. The downside of this approach is that it is somewhat arbitrary (due to the cut-off) and that it may introduce large amounts of shot noise in the particle loss signal.

With the addition of a strong-strong beam-beam functionality, one has to use completely populated bunches. Still, the dynamics of selected sections of phasespace can be better resolved by choosing a distribution

$$
\rho(\xi)=\Theta(1-r)\left(r^{5-\gamma}\right)\left(r^{\gamma} \exp \left(-r^{2} / 2\right)\right)\left(\mathrm{d}\left(r^{6-\gamma}\right) \mathrm{d} \Omega\right)
$$

in normalized phasespace, where $r^{2}=\sum_{i} \xi_{i}^{2}$ and the three factors are contributed by rejection, weighting, and redistribution of a unit qausirandom number distribution. The distribution is filled systematically using a Halton sequence, reducing statistical noise. By adjusting $\gamma$, the resolution can be selectively increased at a chosen action value.

\section{BEAM DYNAMICS}

\section{Chromatic Lumping}

Computational speed requirements may necessitate the lumping of adjacent lattice elements to a single one. A typical setup would lump elements between sextupoles and beam-beam elements. Sextupole elements in dispersive sections will introduce chromaticity, which consequently has to be considered in the lumped sections to obtain the correct total chromaticity of the ring. A common procedure is to insert a $p_{t}$-dependent tune shift after a lumped section, which has the disadvantages of omitting the chromatic changes of the Twiss functions and being non-symplectic. Also, it is not easily applied to arbitrary coupling or to beamline sections. Plibb uses an approach avoiding these drawbacks.

The full map $\mathscr{M}$ of a beamline section will be symplectic around any phase-space vector $x_{0},\left.\partial_{x} \otimes \partial_{x} \mathscr{M}\left(x_{0}+x\right)\right|_{x=0} \in$ $S p(2 n, \mathbb{R})$. While the one-turn map of a real storage ring around the orbit will also be strongly stable, an section's 
map may not even be stable. The transfer map can be expanded in a Taylor map around the orbit:

$$
x_{i}^{\prime}=M_{(0), i}+M_{(1), i k} x^{k}+1 / 2 M_{(2), i k l} x^{k} x^{l} \ldots,
$$

where the orbit has been chosen $x_{0}=0$.

A particle with momentum offset $p_{t}=\Delta$ will follow the dispersion trajectory with an offset of $D_{\Delta}$ with respect to the orbit at the section's entrance; expanding around $D_{\Delta}$ gives a transformation with $\mathrm{O}(\Delta)$ coefficients $\bar{M}_{(1), i k}=$ $M_{(1), i k}+\Delta M_{(2), i k l} D_{\Delta}^{l}$. Expanding the symplecticity condition for the transformation around the $D_{\Delta}$-shifted orbit to first order in $\Delta$ gives

$$
\bar{M}_{(1)}^{\top} J M_{(1)}=M_{(1)}^{\top} J \bar{M}_{(1)}+\mathrm{O}\left(\Delta^{2}\right) \quad ;
$$

plugging $\mathbb{I}+\Delta \bar{G}=M_{(1)}^{\mu-1} \bar{M}_{(1)} M_{(1)}^{-\mu}, \mu \in \mathbb{R}$ into (2) gives $\bar{G}^{\top} J=J \bar{G}+\mathrm{O}\left(\Delta^{2}\right)$, i. e., $\bar{G}$ is a generator of a symplectic transformation, $\bar{G} \in \operatorname{sp}(2 n, \mathbb{R})$, which can be seen by putting $\mu=0$ and using that $\bar{G}$ is a vector of the adjoint representation of the symplectic group.

As the lumping procedure is to incorporate only transverse dynamics and chromaticity, $\mathscr{M}$ will not change $p_{t}$. Projecting out the $\left(t, p_{t}\right)$ plane by a projector $P$ by $G=$ $P \bar{G} P$,

$$
(1+\Delta G)+O\left(\Delta^{2}\right)=e^{\Delta G}=e^{\mathscr{L}\left[p_{t} x^{\top} H x\right]}
$$

where $\mathscr{L}[A]=\{A, \cdot\}$ is the Liouville operator and $H=$ $P H P=-1 / 2 J G$ is a symmetric matrix with zeros in the $t, p_{t}$ entries. One thus obtains the mapping

$$
\mathscr{M}_{\text {approx }}=M_{(1)}^{\mu-1} \exp \mathscr{L}\left[p_{t} x^{\top} H x\right] M_{(1)}^{-\mu}
$$

which is symplectic by construction and agrees with the exact mapping $\mathrm{O}\left(p_{t}^{2}\right)$. One will usually choose $\mu \in\{0,1\}$.

To explicitly construct (4), one brings (3) into blockdiagonal form by constructing appropriate linear combinations of $G$ 's eigenvectors. As $G$ can be any symplectic generator, it can have any admissible eigenvalue structure. The eigenvalues of $G$ will be decomposable in $m$ equivalence sets of cardinality $n_{i}, i \in\{1, \ldots, m\}$, the equivalence relation being the equality of the unique representative $\Lambda=|\Re \lambda|+i|\mathfrak{I} \lambda|$.

Let $\Lambda_{\sigma_{1} \sigma_{2}}=\sigma_{1} \Re \Lambda+\sigma_{2} \mathfrak{I} \Lambda$ and $\left|\Lambda_{\sigma_{1} \sigma_{2}}\right\rangle$ the associated eigenvector. With $\left\langle\Lambda_{\sigma_{1} \sigma_{2}}|:=| \Lambda_{\sigma_{1} \sigma_{2}}\right\rangle^{\top} J$ and for any $a, b \in$ $\mathbb{C}^{2 n}, G \in \operatorname{sp}(n, \mathbb{R})$

$$
\begin{aligned}
\langle a \mid b\rangle & =-\langle b \mid a\rangle \\
\langle a|G| b\rangle & =\langle b|G| a\rangle .
\end{aligned}
$$

Also, for eigenvalues and eigenvectors,

$$
\begin{aligned}
\Lambda_{\sigma_{1}, \sigma_{2}} & =\Lambda_{\sigma_{1},-\sigma_{2}}^{*} \\
\left|\Lambda_{\sigma_{1}, \sigma_{2}}\right\rangle & =\left|\Lambda_{\sigma_{1},-\sigma_{2}}\right\rangle^{*} .
\end{aligned}
$$

and

$$
\Lambda \neq \Lambda^{\prime} \rightarrow\left\langle\Lambda_{\sigma_{1} \sigma_{2}} \mid \Lambda_{\sigma_{3} \sigma_{4}}^{\prime}\right\rangle=0
$$

and

$$
\left|\sigma_{1}+\sigma_{3}\right|+\left|\sigma_{2}+\sigma_{4}\right| \neq 0 \rightarrow\left\langle\Lambda_{\sigma_{1} \sigma_{2}} \mid \Lambda_{\sigma_{3} \sigma_{4}}\right\rangle=0 .
$$

Vectors of equal $\Lambda$ will be linearly combined into sets of real vectors $\left|q_{i_{i}}\right\rangle,\left|p_{i_{i}}\right\rangle, i_{i} \in\left\{N_{i}+1, \ldots, N+n_{i}\right\}, N_{i}=$ $\sum_{k=1}^{i-1} n_{i}$. Introducing generalized coordinates $\left|\xi_{2 i}\right\rangle=$ $\left|q_{i}\right\rangle,\left|\xi_{2 i+1}\right\rangle=\left|p_{i}\right\rangle$, a matrix $B$ can be formed by lining up column vectors $|\xi\rangle$. The matrix $B$ is symplectic, $B^{\top} J B=$ $J$. To prove this, it is sufficient (because of (5) and (7)) to check that $\left\langle p_{i} \mid q_{k}\right\rangle=-\delta_{i k}$ within each of the possible equivalence sets. From the group properties, one obtains the following list of equivalence sets, generator's eigenvalues and the associated normalization prescriptions, real $|p\rangle,|q\rangle$ pairs, and hamiltonian matrices $\tilde{H}_{i k}=-1 / 2 J \tilde{B}=$ $-1 / 2 B^{\top} J G B=-1 / 2\left\langle\xi_{i}|G| \xi_{k}\right\rangle:$

- ('Singlet') $\Lambda_{00}=0$, the finite transformation is the identity mapping.

- (Real pair) $\Lambda_{+0}, \Lambda_{-0} ;\left\langle\Lambda_{+0} \mid \Lambda_{-0}\right\rangle=1$

$$
\begin{aligned}
|q\rangle & =\sqrt{1 / 2}\left(\left|\Lambda_{-0}\right\rangle+\left|\Lambda_{+0}\right\rangle\right) \\
|p\rangle & =\sqrt{1 / 2}\left(\left|\Lambda_{-0}\right\rangle-\left|\Lambda_{+0}\right\rangle\right) \\
H & =\Lambda\left(p^{2}-q^{2}\right) / 2,
\end{aligned}
$$

describing a repulsive, harmonic 'oscillator'.

- (Complex pair:) $\Lambda_{0+}, \Lambda_{0_{-}} ;\left\langle\Lambda_{0+} \mid \Lambda_{0_{-}}\right\rangle=-i$

$$
\begin{aligned}
|q\rangle & =\sqrt{1 / 2}\left(\left|\Lambda_{0-}\right\rangle+\left|\Lambda_{0+}\right\rangle\right) \\
|p\rangle & =i \sqrt{1 / 2}\left(\left|\Lambda_{0-}\right\rangle-\left|\Lambda_{0+}\right\rangle\right) \\
H & =|\Lambda|\left(p^{2}+q^{2}\right) / 2
\end{aligned}
$$

describing a harmonic oscillator.

- (Quadruplet:) $\Lambda_{++}, \Lambda_{+-}, \Lambda_{-+}, \Lambda_{--} ;\left\langle\Lambda_{++} \mid \Lambda_{--}\right\rangle=$ $\left\langle\Lambda_{+-} \mid \Lambda_{-+}\right\rangle^{*}=1$

$$
\begin{aligned}
\left|q_{1}\right\rangle & =1 / 2\left(\left|\Lambda_{--}\right\rangle-\left|\Lambda_{++}\right\rangle+\left|\Lambda_{-+}\right\rangle-\left|\Lambda_{+-}\right\rangle\right) \\
\left|p_{1}\right\rangle & =-1 / 2\left(\left|\Lambda_{++}\right\rangle+\left|\Lambda_{--}\right\rangle+\left|\Lambda_{+-}\right\rangle+\left|\Lambda_{-+}\right\rangle\right) \\
\left|q_{2}\right\rangle & =i / 2\left(\left|\Lambda_{--}\right\rangle+\left|\Lambda_{++}\right\rangle-\left|\Lambda_{-+}\right\rangle-\left|\Lambda_{+-}\right\rangle\right) \\
\left|p_{2}\right\rangle & =i / 2\left(\left|\Lambda_{++}\right\rangle-\left|\Lambda_{--}\right\rangle-\left|\Lambda_{+-}\right\rangle+\left|\Lambda_{-+}\right\rangle\right) \\
H & =\mathfrak{R} \Lambda \sum_{i}\left(p_{i}^{2}-q_{i}^{2}\right) / 2+\mathfrak{I} \Lambda\left(q_{1} p_{2}-q_{2} p_{1}\right)
\end{aligned} .
$$

By setting $\omega^{2}=\mathfrak{R} \Lambda, \Omega=\mathfrak{I} \Lambda, p=m^{3 / 2}\left(v+\Omega e_{3} \times\right.$ $x) / \omega, q=\omega m^{-1 / 2} x$ this can be identified as an isotropic, repulsive oscillator rotating with frequency $-\Omega$ in the $x$-plane.

\section{Implementation}

Degeneracy A complication not treated above is the occurrence of degeneracy: in the spectrum of $G$, an eigenvalue $\Lambda$ may occur with degeneracy $d$. As numerical eigensolvers will return a (linear independent) set of vectors $\left\{\left|\hat{\Lambda}_{\sigma_{1} \sigma_{2}}^{(i)}\right\rangle \mid i=1, \ldots, d\right\}$ spanning the eigenspace, a set 
$\left\{\left|\Lambda_{\sigma_{1} \sigma_{2}}^{(i)}\right\rangle \mid i=1, \ldots, d\right\}$ has to be constructed fulfilling (7). This can be achieved by a generalization of the GramSchmidt orthogonalization to the symplectic case; it requires a case distinction similar to the one given above.

Exact Solutions With $D(\phi)=\left(\begin{array}{cc}\cosh \phi & \sinh \phi \\ \sinh \phi & \cosh \phi\end{array}\right)$, $F(\phi)=\left(\begin{array}{cc}\cos \phi & \sin \phi \\ -\sin \phi & \cos \phi\end{array}\right)$, the closed solutions to the cases above are

$$
\begin{aligned}
& \xi^{\prime}=\xi \\
& \xi^{\prime}=D(\sqrt{|\Lambda|}) \xi \\
& \xi^{\prime}=F(\sqrt{|\Lambda|}) \xi \\
& \xi^{\prime}=F\left(\mathfrak{I} \Lambda \mathbb{I}_{2}\right) D\left(\sqrt{\mathfrak{R} \Lambda} \mathbb{I}_{2}\right) F\left(-\mathfrak{I} \Lambda \mathbb{I}_{2}\right) \xi
\end{aligned}
$$

respectively. As usually $\Delta \ll 1$, a thin-lens approximation is admissible and will be desirable for speed reasons. As a result of our choice of basis, each hamiltonian separates into two parts, each quadratic in coordinates or momenta. This immediately allows us to approximate the associated finite transformation by the kicks associated with both parts.

\section{New Beamline Elements, Wire Compensation}

While originally conceived for the Tevatron, Plibb is now applied to RHIC. All beamline elements occuring in the RHIC beamline definition have been implemented, most of them in an exact and a thin-lens approximations. Plibb supports sector bends, quads, sextupoles, general multipoles, RF cavities, correctors, rotators, electrostatic separators, and general linear elements. All elements are implemented as compile-time polymorphic functors, allowing for differential-algebraic methods (see above).

There is on-going set of experiments at RHIC being performed to evaluate the feasibility of a current-wire compensation scheme for long-range parasitic beam-beam interactions at LHC. Plibb is among the codes [2] being used to simulate the experiments. A wire compensator has been added to Plibb's library of transformation functors; it is implemented using a simple kick scheme. Its effects in the RHIC lattices have been verified by comparison with anaytic results.

Experimentally, the compensation scheme proper has not been checked with RHIC's current setup, as the gold operation does not exhibit significant beam-beam effects. Tune shift and the experimental signatures of current and wire distance scans have been established and compared to simulations codes [2].

\section{Weak-Strong Beam-Beam}

Plibb incorporates several models for the weak-strong beam-beam interaction. They employ different simplifications, starting from $4 \times 4$ dynamics due to a strong beam ellipsoid aligned with the $x, y$ axes to an arbitrary oriented strong beam ellipsoid, including the correct longitudinal dynamics [1] and considering phase-advance effects by slicing the strong beam ellipsoid into equal-weights longitudinal portions. The model does not yet, however, take into account non-drift particle motion for advancing between kicks by the slices.

We also have implemented different approximations for the numeric calculation of the analytic expression for the beam-beam kick, currently, they have to be selected at compile time.

\section{Strong-Strong Beam-Beam}

The most significant change has been the integration of the strong-strong code NIMZOVICH into Plibb. NIMZOVICH was originally designed as a strong-strong code for the simulation of electron machines dominated by a single interaction point; consequently, not much emphasis was laid on the symplectic and higher-order correctness of the lattice. By making it part of Plibb framework, this has been remedied. Also, the code now uses a parallelization scheme optimized for many parasitic crossings and small synchrotron tunes. This allows it to scale well to thousands of processors, making close-to-realistic LHC simulations feasible.

A remaining open question is the behavior of a PIC code such as NIMZOVICH in the context of hadronic simulations with their high demand on low noise and symplectic correctness. While a $4 \times 4$ PIC algorithm for strongstrong beam-beam can be easily seen to be symplectic with an appropriately chosen field solver, the fully coupled and logitudinally interpolating $6 \times 6$ case requires more careful analysis. Also, the contribution of field noise-inherent to PIC methods-to the total diffusion speed of the system needs to be analyzed, possibly complicated by the use of weighted-particle method.

\section{Benchmarking}

Considerable effort has gone into checking the correctness of the transformation functors. For magnetic lattices, we have cross-checked tunes, chromaticities, and lattice funtions against MAD-X results for the full RHIC lattice and MAD8 results for the full Tevatron lattice; in all cases, the agreement was excellent. The chromatic lumping algorithm was checked by comparing chromaticities and tunes of lumpings of various coarseness with one another. Beambeam effects have been checked by comparing tune shifts and tune footpints with analytically known results. Typical results for loss rate estimates for the RHIC experiment are given in [2].

\section{REFERENCES}

[1] K. Hirata, H. Moshammer and F. Ruggiero, Particle Accelerators 40, 205 (1993)

[2] W. Fischer et al., Experiments With a DC Wire in RHIC, This Conference 